J. Nonlinear Var. Anal. 2 (2018), No. 1, pp. 3-23

Available online at http://jnva.biemdas.com

https://doi.org/10.23952/jnva.2.2018.1.02

\title{
STRONGLY NONLINEAR SECOND ORDER MULTIVALUED DIRICHLET SYSTEMS
}

\author{
SERGIU AIZICOVICI ${ }^{1, *}$, NIKOLAOS S. PAPAGEORGIOU $^{2}$, VASILE STAICU $^{3}$ \\ ${ }^{1}$ Department of Mathematics, Ohio University, Athens, OH 45701, USA \\ ${ }^{2}$ Department of Mathematics, National Technical University, Zografou Campus, Athens 15780, Greece \\ ${ }^{3}$ CIDMA - Center for Research and Development in Mathematics and Applications, \\ Department of Mathematics, University of Aveiro, 3810-193 Aveiro, Portugal
}

\begin{abstract}
We consider second order nonlinear Dirichlet systems driven by a nonlinear nonhomogeneous differential operator. The reaction term consists of a maximal monotone map $A(\cdot)$ plus a multivalued perturbation $F$ depending also on the derivative. Using tools from multivalued analysis and from the theory of nonlinear operators of monotone type, we prove existence theorems both for the "convex" ( $F$ convex-valued) and the "nonconvex" ( $F$ nonconvex-valued) problems. We also present an example of a system with unilateral constraints.
\end{abstract}

Keywords. Maximal monotone operator; $p$-Laplace-like operator; Yosida approximation; Vector Sobolev space.

2010 Mathematics Subject Classification. 34B15, 34C25, 47H06.

\section{INTRODUCTION}

In this paper, we study the following second order nonlinear differential inclusion

$$
\left\{\begin{array}{l}
\left(a\left(u^{\prime}(t)\right)\right)^{\prime} \in A(u(t))+F\left(t, u(t), u^{\prime}(t)\right) \text { for a.a. } t \in T:=[0, b], \\
u(0)=u(b)=0 .
\end{array}\right.
$$

In this problem, $a: \mathbb{R}^{N} \rightarrow \mathbb{R}^{N}$ is a monotone homeomorphism, which includes as a special case the vector $p$-Laplacian, $A: \mathbb{R}^{N} \rightarrow 2^{\mathbb{R}^{N}}$ is a maximal monotone map and $F: T \times \mathbb{R}^{N} \rightarrow 2^{\mathbb{R}^{N}} \backslash\{\varnothing\}$ is a set-valued nonlinearity.

Analogous second order systems were studied by Aizicovici-Papageorgiou-Staicu [1], Erbe-Krawcewicz [5], Frigon [6], Frigon-Montoki [7], Halidias-Papageorgiou [10], Kyritsi-Matzakos-Papageorgiou [12], Manasevich-Mawhin [13], Pruszko [16]. In all the above works either $A \equiv 0$ or the multifunction $F$ is independent of $u^{\prime}$ or the conditions on $F$ are more restrictive. The presence of the map $A(\cdot)$ in our problem and the fact that in general $D(A) \neq \mathbb{R}^{N}$, enables us to incorporate in our framework differential variational inequalities.

Our approach uses tools and results from multivalued analysis and from the theory of nonlinear operators of monotone type. In the next section, for the convenience of the reader, we recall the basic definitions and facts from these theories which we will use in the sequel.

\footnotetext{
${ }^{*}$ Corresponding author.
}

E-mail addresses: aizicovs@ ohio.edu (S. Aizicovici), npapg@math.ntua.gr (N.S. Papageorgiou), vasile@ua.pt (V. Staicu).

Received July 24, 2017; Accepted August 28, 2017. 


\section{Mathematical BACKGRound-Hypotheses.}

We start with multivalued analysis. Further details can be found in Hu-Papageorgiou [11]. So, let $(\Omega, \Sigma)$ be a measurable space and $(X,\|\|$.$) be a separable Banach space. We will use the following$ notation:

$$
\begin{gathered}
\mathscr{P}_{f(c)}(X)=\{A \subseteq X: A \text { is nonempty, closed, (and convex) }\}, \\
\mathscr{P}_{(w) k(c)}(X)=\{A \subseteq X: A \text { is nonempty, (weakly-) compact, (and convex) }\} .
\end{gathered}
$$

We will use the symbol $\stackrel{w}{\longrightarrow}$ to designate weak convergence.

For a multifunction (set-valued map) $F: \Omega \rightarrow 2^{X} \backslash\{\varnothing\}$, the graph of $F$ is the set

$$
\operatorname{Gr} F=\{(\omega, x) \in \Omega \times X: x \in F(\omega)\} .
$$

We say that $F($.$) is graph measurable if \operatorname{Gr} F \in \Sigma \times \mathscr{B}(X)$ where $\mathscr{B}(X)$ is the Borel $\sigma$-field of $X$.

If $F($.$) is graph measurable and if \mu$ is a $\sigma$-finite measure on $\Sigma$, then from the Yankov-von NeumannAumann selection theorem (see Hu-Papageorgiou [11], pp.158-159), we know that we can find a sequence of $\Sigma$-measurable selections $f_{n}: \Omega \rightarrow X(n \in \mathbb{N})$ of $F(\cdot)$ such that

$$
F(\omega) \subseteq{\overline{\left\{f_{n}(\omega)\right\}_{n \geq 1}}}_{\text {for } \mu-\text { a.a. } \omega \in \Omega .}
$$

For a $P_{f}(X)$-valued multifunction $F(\cdot)$, we say that $F(\cdot)$ is measurable, if for every $u \in X$, the function

$$
\omega \rightarrow d(u, F(\omega)):=\inf \{\|u-x\|: x \in F(\omega)\}
$$

is $\Sigma$-measurable. For $\mathscr{P}_{f}(X)$-valued multifunctions, measurability implies graph measurability and the converse is true if there is a complete $\sigma$-finite measure $\mu(\cdot)$ on $\Sigma$.

Now suppose that $(\Omega, \Sigma, \mu)$ is a $\sigma$-finite measure space, $1 \leq p \leq \infty$ and $F: \Omega \rightarrow 2^{X} \backslash\{\varnothing\}$ is a multifunction. We define

$$
S_{F}^{p}=\left\{f \in L^{p}(\Omega, X): f(\omega) \in F(\omega) \mu \text {-a.e. }\right\} .
$$

If $F(\cdot)$ is graph measurable, then using the Yankov-von Neumann-Aumann selection theorem, we see that $S_{F}^{p} \neq \varnothing$, if and only if $\omega \rightarrow \inf \{\|x\|: x \in F(\omega)\}$ belongs to $L^{p}(\Omega)$.

The set $S_{F}^{p}$ is decomposable, in the sense that if $\left(A, f_{1}, f_{2}\right) \in \Sigma \times S_{F}^{p} \times S_{F}^{p}$, then

$$
f_{1} \chi_{A}+f_{2} \chi_{\Omega \backslash A} \in S_{F}^{p} .
$$

Here, for any $B \in \Sigma, \chi_{B}$ denotes the characteristic function of $B$.

Let $Y, V$ be Hausdorff topological spaces and $G: Y \rightarrow 2^{V} \backslash\{\varnothing\}$ a multifunction. We say that $G(\cdot)$ is "upper semicontinuous" ("usc" for short) if for every open set $U \subset V, G^{+}(U):=\{y \in Y: G(y) \subset U\}$ is open. $G(\cdot)$ is said to be "lower semicontinuous" ("lsc" for short) if for every open set $U \subset V, G^{-}(U):=$ $\{y \in Y: G(y) \cap U \neq \varnothing\}$ is open.

If $V$ is regular space, then an usc multifunction has closed graph. The converse is true if $G$ has closed values and is locally compact (that is, for every $y \in Y$ we can find $U$, a neighborhood of $y$ such that $\overline{G(U)}$ is compact in $V$ ). Also, if $V$ is a metric space, then a $\mathscr{P}_{f}(V)$-valued multifunction $G: Y \rightarrow$ $2^{V} \backslash\{\varnothing\}$ is lsc if and only if for all $v \in V$, the function $y \rightarrow d(v, G(y)):=\inf \{\|v-x\|: x \in G(y)\}$ is upper semicontinuous.

Now we present some basic definitions and facts for nonlinear operators of monotone type. For further details we refer to Gasinski-Papageorgiou [8]. So, let $X$ be a reflexive Banach space and $X^{*}$ its topological 
dual. By $\langle\cdot, \cdot\rangle$ we denote the duality brackets for the pair $\left(X^{*}, X\right)$. A multivalued map $A: X \rightarrow 2^{X^{*}}$ is said to be monotone if for all $\left(x, x^{*}\right),\left(u, u^{*}\right) \in G r A$, we have

$$
\left\langle u^{*}-x^{*}, u-x\right\rangle \geq 0 \text {. }
$$

If $\left\langle u^{*}-x^{*}, u-x\right\rangle=0 \Longrightarrow x=u$, then we say that $A$ is strictly monotone. The set

$$
D(A):=\left\{x \in \mathbb{R}^{N}: A(x) \neq \varnothing\right\}
$$

is the domain of $A$. We say that a monotone map $A(\cdot)$ is maximal monotone if

$$
\left[\left\langle u^{*}-x^{*}, u-x\right\rangle \geq 0 \text { for all }\left(x, x^{*}\right) \in G r A\right] \Longrightarrow\left(u, u^{*}\right) \in G r A,
$$

that is, $\mathrm{Gr} A$ is not properly contained in the graph of a monotone map.

Let $X_{w}$ (resp. $X_{w}^{*}$ ) denote the space $X$ (resp. $X^{*}$ ) endowed with the weak topology. We can easily see that for a maximal monotone map $A: X \rightarrow 2^{X^{*}}, G r A$ is closed in $X_{w} \times X^{*}$ and in $X \times X_{w}^{*}$.

Let $X=H$ be a Hilbert space and identify $H$ with its dual by the Riesz-Frechet theorem (that is $\left.H=H^{*}\right)$. Let $A: H \rightarrow 2^{H}$ be a maximal monotone map.

For $\lambda>0$, we define the following single valued maps

$$
\begin{aligned}
& \left.J_{\lambda}:=(I+\lambda A)^{-1} \text { (the resolvent of } A\right), \\
& \left.A_{\lambda}:=\frac{1}{\lambda}\left(I-J_{\lambda}\right) \text { (the Yosida approximation of } A\right) .
\end{aligned}
$$

The next proposition summarizes the properties of these two operators.

Proposition 2.1. If $A: H \rightarrow 2^{H}$ is a maximal monotone map and $\lambda>0$, then:

(a) $J_{\lambda}: H \rightarrow H$ is nonexpansive (that is, Lipschitz continuous with Lipschitz constant 1 );

(b) $A_{\lambda}(x) \in A\left(J_{\lambda}(x)\right)$ for all $x \in H$;

(c) $A_{\lambda}($.$) is monotone and Lipschtz continuous with Lipschitz constant \frac{1}{\lambda}$ (therefore $A_{\lambda}($.$) is maxi-$ mal monotone too);

(d) $\left\|A_{\lambda}(x)\right\| \leq\left\|A^{0}(x)\right\|=\min \left\{\left\|x^{*}\right\|: x^{*} \in A(x)\right\}$ and $A_{\lambda}(x) \rightarrow A^{0}(x)$ as $\lambda \rightarrow 0^{+}$for all $x \in D(A)$;

(e) $\overline{D(A)}$ is convex and $J_{\lambda}(x) \rightarrow p_{\overline{D(A)}}(x)$ as $\lambda \rightarrow 0^{+}$for all $x \in H$.

Remark 2.1. The maximal monotonicity of $A(\cdot)$ implies that for all $x \in D(A), A(x) \in P_{f c}(H)$ and so $A^{0}(x)$ is well defined as the element of minimal norm in $A(x)$ (see Proposition $2.1(d)$ ). Also $p_{\overline{D(A)}}(\cdot)$ denotes the metric projection on the closed convex set $\overline{D(A)}$ (see Proposition $2.1(e)$ ). According to part $(e), J_{\lambda}($.$) can be viewed as an approximation of the identity map. Note that if D(A)=H$, then $J_{\lambda}(x) \rightarrow x$ for all $x \in H$.

Let $Y, V$ be two Banach spaces and $K: Y \rightarrow V$. We say that:

(a) $K$ is completely continuous, if

$$
y_{n} \stackrel{w}{\longrightarrow} y \text { in } Y \Longrightarrow K\left(y_{n}\right) \rightarrow K(y) \text { in } V .
$$

(b) $K$ is compact, if it is continuous and maps bounded sets into relatively compact sets. 
In general, these two notions are distinct. However, if $Y$ is reflexive, then "complete continuity" implies "compactness". If $Y$ is reflexive and $K \in \mathscr{L}(Y, V)$, then "complete continuity" and "compactness" of $K$ are equivalent.

A multifunction $G: Y \rightarrow 2^{V} \backslash\{\varnothing\}$ is said to be compact if it is usc and maps bounded subsets of $Y$ into relatively compact subsets of $V$.

Suppose $G: Y \rightarrow \mathscr{P}_{w k c}(V)$ is upper semicontinuous from $Y$ into $V_{w}, K: V \rightarrow Y$ is completely continuous and $Q=K \circ G$. The following generalization of the Leray-Schauder Alternative Theorem (see [9], Theorem 4.93, p. 642), is due to Bader [2].

Proposition 2.2. If $Y, V, Q$ are as above and $Q$ is compact, then one and only one of the following properties holds:

(a) $S:=\{y \in Y: y \in t Q(y)$ for some $0<t<1\}$ is unbounded;

(b) $Q(\cdot)$ has a fixed point.

In what follows, by $|\cdot|$ we denote the norm of $\mathbb{R}^{N}$ and by $(\cdot, \cdot)_{\mathbb{R}^{N}}$ we denote the usual inner product on $\mathbb{R}^{N}$. By $\|\cdot\|$ we denote the norm of the Sobolev space $W_{0}^{1, p}\left((0, b) ; \mathbb{R}^{N}\right)$ and by $\|\cdot\|_{p}$ we denote the norm of the space $L^{p}\left((0, b) ; \mathbb{R}^{N}\right)$. From the Poincare inequality, we have

$$
\|u\|=\left\|u^{\prime}\right\|_{p} \text { for all } u \in W_{0}^{1, p}\left((0, b) ; \mathbb{R}^{N}\right), 1<p<\infty .
$$

Recall that

$$
\left(W_{0}^{1, p}\left((0, b) ; \mathbb{R}^{N}\right)\right)^{*}=W^{-1, p^{\prime}}\left((0, b) ; \mathbb{R}^{N}\right), \text { where } \frac{1}{p}+\frac{1}{p^{\prime}}=1 .
$$

By $\langle\cdot, \cdot\rangle$ we denote the duality brackets for this dual pair.

In the sequel, for notational simplicity, we write $W_{0}^{1, p}$ for $W_{0}^{1, p}\left((0, b) ; \mathbb{R}^{N}\right), W^{-1, p^{\prime}}$ for

$$
W^{-1, p^{\prime}}\left((0, b) ; \mathbb{R}^{N}\right)=\left(W_{0}^{1, p}\left((0, b) ; \mathbb{R}^{N}\right)\right)^{*}
$$

(with $\frac{1}{p}+\frac{1}{p^{\prime}}=1$ ), and $L^{q}$ for $L^{q}\left(T, \mathbb{R}^{N}\right)$, where $T=[0, b]$ and $1 \leq q \leq \infty$.

We will also need some information about the spectrum of the vector Dirichlet $p$-Laplacian. So, we consider the following nonlinear vector eigenvalue problem

$$
\left\{\begin{array}{l}
\left(-\left|u^{\prime}(t)\right|^{p-2} u^{\prime}(t)\right)^{\prime}=\widehat{\lambda}|u(t)|^{p-2} u(t) \text { for a.a. } t \in T:=[0, b], \\
u(0)=u(b)=0,
\end{array}\right.
$$

where $1<p<\infty$. We know (see Gasinski-Papageorgiou [8], Theorem 6.3.10, p.768) that (2.1) has a sequence of eigenvalues $\left\{\widehat{\lambda}_{n}\right\}_{n \geq 1} \subset(0,+\infty)$ such that

$$
\widehat{\lambda}_{n}=\left(\frac{n}{b}\right)^{p}(p-1)\left[2 \int_{0}^{1} \frac{d t}{\left(1-t^{p}\right)^{\frac{1}{p}}}\right]^{p} \text { for all } n \geq 1 .
$$

So, $\widehat{\lambda}_{n} \rightarrow+\infty$ as $n \rightarrow+\infty$. The corresponding eigenfunctions are

$$
\widehat{u}_{n}(t)=\xi u_{n}(t) \text { for all } t \in T, \text { all } n \geq 1,
$$


where $\xi \in \mathbb{R}^{N}$ and $u_{n}(\cdot)$ is the corresponding scalar eigenfunction. Also recall the following variational characterization of $\widehat{\lambda}_{1}$ (see [8], p. 761)

$$
\widehat{\lambda}_{1}=\inf \left\{\frac{\left\|u^{\prime}\right\|_{p}^{p}}{\|u\|_{p}^{p}}: u \in W_{0}^{1, p}(0, b), u \neq 0\right\} .
$$

These facts lead to the following useful result (see Motreanu-Motreanu-Papageorgiou [15], Lemma 11.3, p.305):

Proposition 2.3. If $\eta \in L^{\infty}(T), \eta(t) \leq \widehat{\lambda}_{1}$ for a.a. $t \in T$ and $\eta \neq \widehat{\lambda}_{1}$, then there exists $C_{1}>0$ such that

$$
\left\|u^{\prime}\right\|_{p}^{p}-\int_{0}^{b} \eta(t)|u(t)|^{p} d t \geq C_{1}\|u\|^{p} \text { for all } u \in W_{0}^{1, p} .
$$

Now we introduce our hypotheses on the data of (1.1).

$\mathbf{H}(a): a: \mathbb{R}^{N} \rightarrow \mathbb{R}^{N}$ is of the form $a(x)=k(x) x$ or $a(x)=\left(k_{m}(x) x_{m}\right)_{m=1}^{N}$ with $k: \mathbb{R}^{N} \rightarrow \mathbb{R}^{+}$or $k_{m}:$ $\mathbb{R} \rightarrow \mathbb{R}^{+}$continuous and such that

(i) $a(\cdot)$ is strictly monotone;

(ii) $(a(x), x)_{\mathbb{R}^{N}} \geq C_{0}|x|^{p}$ for all $x \in \mathbb{R}^{N}$ some $C_{0}>0$ and with $p \geq 2$.

Remark 2.2. These conditions on $a(\cdot)$ are a little more restrictive than those employed by ManasevichMawhin [13]. However, they are general enough to incorporate in our analysis many differential operators of interest. For example, let

$$
a(x)=|x|^{p-2} x \text { or } a(x)=\left(\left|x_{m}\right|^{p-2} x_{m}\right)_{m=1}^{N} \text { for all } x=\left(x_{m}\right)_{m=1}^{N} \in \mathbb{R}^{N}, p \geq 2 .
$$

Then these maps satisfy hypotheses $\mathbf{H}(a)$ and correspond to different versions of the $p$-Laplacian (see Zhang [17]). We stress that we do not impose any growth conditions on $a(\cdot)$. So, if $\beta(x)=\frac{1}{p}\left(C e^{|x|^{p}}-1\right)$ with $C>1,2 \leq p<\infty$, then

$$
a(x)=\nabla \beta(x)=\left(C e^{|x|^{p}}-1\right)|x|^{p-2} x, x \in \mathbb{R}^{N}
$$

satisfies hypotheses $\mathbf{H}(a)$. Another possibility for $a(\cdot)$ is

$$
a(x)=\xi(|x|)|x|^{p-2} x, p \geq 2,
$$

with $\xi: \mathbb{R}^{+} \rightarrow \mathbb{R}^{+}$continuous, $0<C_{0} \leq \xi(r)$ for all $r \geq 0$ and $r \rightarrow \xi(r) r^{p-1}$ strictly increasing on $(0,+\infty)$. For example, let $\xi(r)=\frac{r+1}{r+2}$.

Note that $a(\cdot)$ is a homeomorphism and $\left|a^{-1}(x)\right| \rightarrow+\infty$ as $|x| \rightarrow+\infty$.

$\mathbf{H}(A): A: \mathbb{R}^{N} \rightarrow 2^{\mathbb{R}^{N}}$ is a maximal monotone map such that $0 \in A(0)$.

Remark 2.3. We stress that we do not assume that $D(A)=\mathbb{R}^{N}$. This way we incorporate in our analysis differential variational inequalities (that is, systems with unilateral constraints).

$\mathbf{H}(F): F: T \times \mathbb{R}^{N} \times \mathbb{R}^{N} \rightarrow \mathscr{P}_{k c}\left(\mathbb{R}^{N}\right)$ is a multifunction such that

(i) for a. a. $x, y \in \mathbb{R}^{N}, t \rightarrow F(t, x, y)$ is graph measurable;

(ii) for a. a. $t \in T,(x, y) \rightarrow F(t, x, y)$ has closed graph;

(iii) for a.a. $t \in T$, all $x, y \in \mathbb{R}^{N}$ and all $v \in F(t, x, y)$, we have

$$
|v| \leq \gamma_{1}(t,|x|)+\gamma_{2}(t,|x|)|y|^{p-1},
$$


where $\gamma_{i}: T \times \mathbb{R}^{+} \rightarrow \mathbb{R}^{+}$satisfy

$$
\sup \left\{\gamma_{1}(t, r): 0 \leq r \leq k\right\} \leq \eta_{1, k}(t), \eta_{1, k} \in L^{p^{\prime}}(T)_{+}
$$

and

$$
\sup \left\{\gamma_{2}(t, r): 0 \leq r \leq k\right\} \leq \eta_{2, k}(t), \eta_{2, k} \in L^{\infty}(T)_{+} ;
$$

(iv) if $m(t, x, y):=\inf \left\{(v, x)_{\mathbb{R}^{N}}: v \in F(t, x, y)\right\}$, then

$$
\liminf _{|x| \rightarrow+\infty} \frac{\inf \left\{m(t, x, y): y \in \mathbb{R}^{N}\right\}}{|x|^{p}} \geq-\theta(t) \text { uniformly for a.a. } t \in T,
$$

where $\theta \in L^{\infty}(T)_{+}, \theta(t) \leq C_{0} \widehat{\lambda}_{1}$ for a.a. $t \in T, \theta \neq C_{0} \widehat{\lambda}_{1}$ with $C_{0}>0$ as in hypothesis $\mathbf{H}(a)(i i)$.

Remark 2.4. Note that $t \rightarrow F(t, x, y)$ is measurable and for a.a. $t \in T, F(t, \cdot, \cdot)$ is usc. Hypothesis $\mathbf{H}(F)(i v)$ is a kind of nonresonance condition. Indeed, if $a(x)=|x|^{p-2} x$ (the vector p-Laplacian operator, hence $\left.C_{0}=1\right), A \equiv 0$ and $F$ is single valued and independent on $y \in \mathbb{R}^{N}$, then hypothesis $\mathbf{H}(F)(i v)$ is a nonuniform nonresonance condition employed quite often in problems of variational character (see Zhang [17]).

\section{Auxiliary RESUlts}

Throughout the remainder of the paper, $p \in[2, \infty)$ is the same as in assumption $\mathbf{H}(a)(i i)$.

We start by considering the following auxiliary Dirichlet system

$$
\left\{\begin{array}{l}
-\left(a\left(u^{\prime}(t)\right)\right)^{\prime}+|u(t)|^{p-2} u(t)=g(t) \text { for a.a. } t \in T:=[0, b], \\
u(0)=u(b)=0 .
\end{array}\right.
$$

with $g \in L^{p^{\prime}}\left(T, \mathbb{R}^{N}\right)$ (recall that $\left.\frac{1}{p}+\frac{1}{p^{\prime}}=1\right)$.

Proposition 3.1. If hypotheses $\mathbf{H}\left(\right.$ a) hold, then problem (3.1) has a unique solution $u \in C^{1}\left(T, \mathbb{R}^{N}\right)$.

Proof. For every $h \in L^{p^{\prime}}\left(T, \mathbb{R}^{N}\right)$, the Dirichlet system

$$
\left\{\begin{array}{l}
-\left(a\left(u^{\prime}(t)\right)\right)^{\prime}=h(t) \text { for a.a. } t \in T:=[0, b], \\
u(0)=u(b)=0
\end{array}\right.
$$

has a unique solution. To see this, note that from (3.2) after integration over $[0, t]$, we obtain

$$
a\left(u^{\prime}(t)\right)=\alpha-H(h)(t) \text { for a.a. } t \in T,
$$

where $\alpha \in \mathbb{R}^{N}$ and $H: L^{p^{\prime}}\left(T, \mathbb{R}^{N}\right) \rightarrow C\left(T, \mathbb{R}^{N}\right)$ is defined by

$$
H(h)(t)=\int_{0}^{t} h(s) d s
$$

So, we have

$$
u^{\prime}(t)=a^{-1}(\alpha-H(h)(t)) \text { for a.a. } t \in T, u(0)=u(b)=0 .
$$

Integrating over $[0, t]$, we obtain

$$
u(t)=\int_{0}^{t} a^{-1}(\alpha-H(h)(s)) d s
$$


Note that $u(b)=\int_{0}^{b} a^{-1}(\alpha-H(h)(s)) d s=0$. From Proposition $2.2(i)$ of Manasevich-Mawhin [13], we know that the equation $\int_{0}^{b} a^{-1}(\alpha-H(h)(s)) d s=0$ in $\alpha \in \mathbb{R}^{N}$, has a unique solution $\alpha=\sigma(H(h))$. Therefore problem (3.2) has a unique solution

$$
u(t)=\int_{0}^{t} a^{-1}(\sigma(H(h))-H(h)(s)) d s .
$$

Now let $K: L^{p^{\prime}} \rightarrow W_{0}^{1, p}$ be the map which to each $h \in L^{p^{\prime}}\left(T, \mathbb{R}^{N}\right)$ assigns the unique solution (3.3) for problem (3.1).

Claim 1: $K: L^{p^{\prime}} \rightarrow W_{0}^{1, p}$ is completely continuous.

Let $h_{n} \stackrel{w}{\longrightarrow} h$ in $L^{p^{\prime}}$ and set $u_{n}=K\left(h_{n}\right) \in W_{0}^{1, p}$ for any $n \in \mathbb{N}$. We have

$$
-\left(a\left(u_{n}^{\prime}(t)\right)\right)^{\prime}=h_{n}(t) \text { for a.a. } t \in T, \text { all } n \in \mathbb{N} .
$$

Taking the inner product with $u_{n}(t)$, integrating over $T=[0, b]$ and performing an integration by parts, we obtain

$$
C_{0}\left\|u_{n}^{\prime}\right\|_{p}^{p} \leq\left\|h_{n}\right\|_{p}\left\|u_{n}\right\|_{p}
$$

(see hypothesis $\mathbf{H}(a)(i i)$ ). Hence $\left\{u_{n}\right\}_{n \geq 1} \subseteq W_{0}^{1, p}$ is bounded (by Poincare's inequality). Also, directly from (3.4) we have that

$$
\left\{\left(a\left(u_{n}^{\prime}(t)\right)\right)^{\prime}\right\}_{n \geq 1} \subseteq L^{p^{\prime}} \text { is bounded. }
$$

From (3.3), we know that

$$
u_{n}^{\prime}(t)=a^{-1}(\alpha-H(h)(t)) \text { for a.a. } t \in T, \text { all } n \in \mathbb{N} .
$$

From Proposition 2.2 (ii) of Manasevich-Mawhin [13] we know that the solution map $\sigma: C\left(T, \mathbb{R}^{N}\right) \rightarrow$ $\mathbb{R}^{N}$ is continuous and bounded (that is, maps bounded sets to bounded sets). Also, $H: L^{p^{\prime}} \rightarrow C\left(T, \mathbb{R}^{N}\right)$ is linear continuous. Moreover, if $N_{1}: C\left(T, \mathbb{R}^{N}\right) \rightarrow C\left(T, \mathbb{R}^{N}\right)$ is defined by

$$
N_{1}(y)(\cdot)=a^{-1}(y(\cdot))
$$

then $N_{1}$ is continuous and maps bounded sets to bounded sets. So, from (3.6) it follows that we can find $C_{2}>0$ such that

$$
\left|u_{n}^{\prime}(t)\right| \leq C_{2} \text { for all } t \in T, \text { all } n \in \mathbb{N}
$$

hence

$$
\left|a\left(u_{n}^{\prime}(t)\right)\right| \leq C_{3} \text { for some } C_{3}>0, \text { all } t \in T, \text { all } n \in \mathbb{N},
$$

therefore

$$
\left\{a\left(u_{n}^{\prime}(\cdot)\right)\right\}_{n \geq 1} \subseteq W^{1, p^{\prime}} \text { is bounded, }
$$

and we conclude that

$$
\left\{a\left(u_{n}^{\prime}(\cdot)\right)\right\}_{n \geq 1} \subseteq C\left(T, \mathbb{R}^{N}\right) \text { is relatively compact, }
$$


(recall that $W^{1, p^{\prime}} \hookrightarrow C\left(T, \mathbb{R}^{N}\right)$ compactly). The continuity of $N_{1}(\cdot)$ implies that $\left\{u_{n}^{\prime}\right\}_{n \geq 1} \subseteq C\left(T, \mathbb{R}^{N}\right)$ is relatively compact. Therefore, we can say that

$$
\left\{u_{n}\right\}_{n \geq 1} \subseteq C^{1}\left(T, \mathbb{R}^{N}\right) \text { is relatively compact. }
$$

So, we may assume that

$$
u_{n} \rightarrow u \text { in } C^{1}\left(T, \mathbb{R}^{N}\right) .
$$

Passing to the limit as $n \rightarrow \infty$ in (3.4) we have

$$
-\left(a\left(u^{\prime}(t)\right)\right)^{\prime}=h(t) \text { for a.a. } t \in T, u(0)=u(b)=0,
$$

hence $u \in K(h)$, therefore $K$ is completely continuous. This proves Claim 1 .

Next, let $N_{2}: W_{0}^{1, p} \rightarrow L^{p^{\prime}}$ be the map defined by

$$
N_{2}(u)(\cdot)=-|u(\cdot)|^{p-2} u(\cdot)+g(\cdot) .
$$

Evidently, $N_{2}(\cdot)$ is continuous and bounded. A solution of (3.1) is a fixed point of the composition $K \circ N_{2}$. We shall produce such a fixed point using the classical Leray-Schauder alternative theorem.

Claim 2: The set $S=\left\{u \in W_{0}^{1, p}: u=\beta\left(K \circ N_{2}\right)(u)\right.$ for some $\left.\beta \in(0,1)\right\}$ is bounded.

Let $u \in S$. We have

$$
-\left(a\left(\frac{1}{\beta} u^{\prime}(t)\right)\right)^{\prime}+|u(t)|^{p-2} u(t)=g(t) \text { for a.a. } t \in T, u(0)=u(b)=0 .
$$

Taking the inner product with $u(t)$, integrating over $T=[0, b]$ and using as before integration by parts, we obtain

$$
\frac{C_{0}}{\beta^{p-1}}\left\|u^{\prime}\right\|_{p}^{p}+\frac{1}{\beta^{p-1}}\|u\|_{p}^{p} \leq\|g\|_{p^{\prime}}\|u\|_{p}
$$

(see hypothesis $\mathbf{H}(a)(i i)$ ), hence

$$
\|u\|^{p-1} \leq C_{4}\|g\|_{p^{\prime}} \text { for some } C_{4}>0
$$

therefore $S \subseteq W_{0}^{1, p}$ is bounded. This proves Claim 2 .

We apply the Leray-Schauder alternative theorem and obtain $\widehat{u} \in C^{1}\left(T, \mathbb{R}^{N}\right)$ such that $\widehat{u}=\left(K \circ N_{2}\right)(\widehat{u})$. Then $\widehat{u} \in C^{1}\left(T, \mathbb{R}^{N}\right)$ is a solution of (3.1). In fact the solution is unique on account of the strictly monotonicity of $\mathbb{R}^{N} \ni x \rightarrow|x|^{p-2} x$.

Consider the operator $V: D(V) \subseteq L^{p} \rightarrow L^{p^{\prime}}$ defined by

$$
V(u)(\cdot)=-a\left(u^{\prime}(\cdot)\right)
$$

for all $u \in D(V)=\left\{u \in C^{1}\left(T, \mathbb{R}^{N}\right): a\left(u^{\prime}(\cdot)\right) \in W^{1, p^{\prime}}, u(0)=u(b)=0\right\}$.

Proposition 3.2. If hypotheses $\mathbf{H}(a)$ hold, then $V: D(V) \subseteq L^{p} \rightarrow L^{p^{\prime}}$ is maximal monotone.

Proof. Let $J: L^{p} \rightarrow L^{p^{\prime}}$ be the continuous, strictly monotone (hence maximal monotone, too) coercive map, defined by

$$
J(u)(\cdot)=|u(\cdot)|^{p-2} u(\cdot) \text { for all } u \in L^{p} .
$$

According to Proposition 3.1, we have

$$
R(V+J)=L^{p^{\prime}}
$$

We show that (3.7) implies the maximal monotonicity of $V(\cdot)$. 
In what follows, let $(\cdot, \cdot)_{p p^{\prime}}$ denote the duality brackets for the dual pairs $\left(L^{p^{\prime}}, L^{p}\right)$, that is,

$$
(h, u)_{p p^{\prime}}=\int_{0}^{b}(h(t), u(t))_{\mathbb{R}^{N}} d t \text { for all } h \in L^{p^{\prime}}, \text { all } u \in L^{p} .
$$

Evidently, $V(\cdot)$ is monotone. Suppose that for some $y \in L^{p}$ and some $v \in L^{p^{\prime}}$ we have

$$
(V(u)-v, u-y)_{p p^{\prime}} \geq 0 \text { for all } u \in D(V) .
$$

Exploiting the surjectivity of $V+J$ (see (3.7)), we can find $u_{1} \in D(V)$ such that

$$
V\left(u_{1}\right)+J\left(u_{1}\right)=v+J(y) .
$$

We use this in (3.8) to obtain

$$
\left(V\left(u_{1}\right)-V\left(u_{1}\right)-J\left(u_{1}\right)+J(y), u_{1}-y\right)_{p p^{\prime}} \geq 0
$$

hence

$$
\left(J\left(u_{1}\right)-J(y), u_{1}-y\right)_{p p^{\prime}} \leq 0,
$$

therefore $y=u_{1} \in D(V)$ and $v=V\left(u_{1}\right)$. This proves the maximality of $V($.$) .$

Now we consider the following approximation of problem (1.1):

$$
\left\{\begin{array}{l}
\left(a\left(u^{\prime}(t)\right)\right)^{\prime} \in A_{\lambda}(u(t))+F\left(t, u(t), u^{\prime}(t)\right) \text { for a.a. } t \in T:=[0, b] \\
u(0)=u(b)=0, \lambda>0
\end{array}\right.
$$

We prove the existence of solutions for problem (3.9).

Proposition 3.3. If hypotheses $\mathbf{H}(a), \mathbf{H}(A), \mathbf{H}(F)$ hold, then problem (3.9) has a solution $u_{\lambda} \in C^{1}\left(T, \mathbb{R}^{N}\right)$.

Proof. Let $\mathscr{A}_{\lambda}: L^{p} \rightarrow L^{p^{\prime}}$ be the Nemitsky operator corresponding to $A_{\lambda}(\cdot)$, that is,

$$
\mathscr{A}_{\lambda}(u)(\cdot)=A_{\lambda}(u(\cdot)) \text { for all } u \in L^{p} .
$$

This map is continuous, monotone, hence maximal monotone. Let $E_{\lambda}=V+J+\mathscr{A}_{\lambda}: D(V) \subseteq L^{p} \rightarrow L^{p \prime}$. Proposition 3.2 and Theorem 3.2.41 of Gasinski-Papageorgiou ([8], p. 328) imply that $E_{\lambda}$ is maximal monotone. Also, for $u \in D(V)$ we have

$$
\begin{aligned}
\left(E_{\lambda}(u), u\right)_{p p^{\prime}} & =(V(u), u)_{p p^{\prime}}+(J(u), u)_{p p^{\prime}}+\left(\mathscr{A}_{\lambda}(u), u\right)_{p p^{\prime}} \\
& \geq C_{0}\left\|u^{\prime}\right\|_{p}^{p}+\|u\|_{p}^{p}
\end{aligned}
$$

(see hypothesis $\mathbf{H}(a)(i i)$ and note that $\mathscr{A}_{\lambda}(0)=0$ ), hence $E_{\lambda}$ is coercive.

But a maximal monotone, coercive map, is surjective (see Gasinski-Papageorgiou ([8], Corollary 3.2.31, p.319). Also $E_{\lambda}$ is strictly monotone (since $J(\cdot)$ is), and so it is injective, therefore $E_{\lambda}$ is a bijection.

Consider the map $E_{\lambda}^{-1}: L^{p \prime} \rightarrow D(V) \subseteq W_{0}^{1, p}$.

Claim 3: $E_{\lambda}^{-1}: L^{p^{\prime}} \rightarrow W_{0}^{1, p}$ is completely continuous.

Let $g_{n} \stackrel{w}{\longrightarrow} h$ in $L^{p^{\prime}}$ and set $u_{n}=E_{\lambda}^{-1}\left(g_{n}\right)$ for all $n \in \mathbb{N}$. We have

$$
E_{\lambda}\left(u_{n}\right)=g_{n} \text { for all } n \in \mathbb{N},
$$


and so

$$
\left\{\begin{array}{l}
-\left(a\left(u_{n}^{\prime}(t)\right)\right)^{\prime}+\left|u_{n}(t)\right|^{p-2} u_{n}(t)+A_{\lambda}\left(u_{n}(t)\right)=g_{n}(t) \text { for a.a. } t \in T \\
u_{n}(0)=u_{n}(b)=0
\end{array}\right.
$$

As before, we take the inner product with $u_{n}(t)$, integrate over $T=[0, b]$, use integration by parts and hypothesis $\mathbf{H}(a)(i i)$, and recall that for all $x \in \mathbb{R}^{N},\left(A_{\lambda}(x), x\right)_{\mathbb{R}^{N}} \geq 0$ (since $\left.A_{\lambda}(0)=0\right)$. So, we obtain

$$
C_{0}\left\|u_{n}^{\prime}\right\|_{p}^{p}+\left\|u_{n}\right\|_{p}^{p} \leq\left\|g_{n}\right\|_{p^{\prime}}\left\|u_{n}\right\|_{p}
$$

hence $\left\{u_{n}\right\}_{n \geq 1} \subseteq W_{0}^{1, p}$ is bounded, therefore $\left\{u_{n}\right\}_{n \geq 1} \subseteq C\left(T, \mathbb{R}^{N}\right)$ is relatively compact (recall that $W_{0}^{1, p} \hookrightarrow C\left(T, \mathbb{R}^{N}\right)$ compactly). Then from (3.10) and Proposition 2.1, we see that

$$
\left\{\left(a\left(u_{n}^{\prime}(\cdot)\right)\right)^{\prime}\right\}_{n \geq 1} \subseteq L^{p^{\prime}} \text { is bounded. }
$$

Also, from (3.10) we have

$$
\left(a\left(u_{n}^{\prime}(t)\right)\right)^{\prime}=\left|u_{n}(t)\right|^{p-2} u_{n}(t)+A_{\lambda}\left(u_{n}(t)\right)-g_{n}(t) \text { for a.a. } t \in T,
$$

hence

$$
\begin{array}{r}
a\left(u_{n}^{\prime}(t)\right)=a\left(u_{n}^{\prime}(0)\right)+\int_{0}^{t}\left[\left|u_{n}(s)\right|^{p-2} u_{n}(s)+A_{\lambda}\left(u_{n}(s)\right)-g_{n}(s)\right] d s \\
\text { for all } t \in T, \text { all } n \in \mathbb{N} .
\end{array}
$$

Set

$$
\widetilde{k}\left(u_{n}\right)(t):=-\left|u_{n}(t)\right|^{p-2} u_{n}(t)-A_{\lambda}\left(u_{n}(t)\right)+g_{n}(t) .
$$

Then $\widetilde{k}\left(u_{n}\right)(\cdot) \in L^{p^{\prime}}$ for all $n \in \mathbb{N}$ and we have

$$
u_{n}^{\prime}(t)=a^{-1}\left(a\left(u_{n}^{\prime}(0)\right)-H\left(\widetilde{k}\left(u_{n}\right)\right)(t)\right), \text { for all } t \in T .
$$

Since $\int_{0}^{b} u_{n}^{\prime}(t) d t=0$ (by the Dirichlet boundary condition), we have

$$
a\left(u_{n}^{\prime}(0)\right)=\sigma\left(H\left(\widetilde{k}\left(u_{n}\right)\right)\right) \text { for all } n \in \mathbb{N}
$$

(see Manasevich-Mawhin [13], Proposition 2.2 (i) and the proof of Proposition 3.1).

Therefore

$$
u_{n}^{\prime}(t)=a^{-1}\left(\sigma\left(H\left(\widetilde{k}\left(u_{n}\right)\right)\right)-H\left(\left(\widetilde{k}\left(u_{n}\right)\right)(t)\right)\right), \text { for all } t \in T
$$

Note that

$$
\left\|H\left(\widetilde{k}\left(u_{n}\right)\right)\right\|_{C\left(T, \mathbb{R}^{N}\right)} \leq C_{5} \text { for some } C_{5}>0, \text { all } n \in \mathbb{N} .
$$

Also, we know that $\sigma: C\left(T, \mathbb{R}^{N}\right) \rightarrow \mathbb{R}^{N}$ is continuous and bounded (i.e., maps bounded sets to bounded sets). Similarly, recall that $N_{1}: C\left(T, \mathbb{R}^{N}\right) \rightarrow C\left(T, \mathbb{R}^{N}\right)$ defined by

$$
N_{1}(u)=a^{-1}(u(\cdot)) \text { for all } u \in C\left(T, \mathbb{R}^{N}\right)
$$

is continuous and bounded. Therefore from (3.11) it follows that we can find $C_{6}>0$ such that

$$
\left|u_{n}^{\prime}(t)\right| \leq C_{6} \text { for all } n \in \mathbb{N} \text {, all } t \in T,
$$

hence

$$
\left|a\left(u_{n}^{\prime}(t)\right)\right| \leq C_{7} \text { for some } C_{7}>0, \text { all } n \in \mathbb{N} \text {, all } t \in T
$$


(recall that $a(\cdot)$ is continuous). So, finally we conclude that $\left\{a\left(u_{n}^{\prime}\right)\right\}_{n>1} \subseteq W^{1, p^{\prime}}$ is bounded, hence $\left\{a\left(u_{n}^{\prime}\right)\right\}_{n \geq 1} \subseteq C\left(T, \mathbb{R}^{N}\right)$ is relatively compact, and we derive that $\left\{u_{n}^{\prime}\right\}_{n \geq 1} \subseteq C\left(T, \mathbb{R}^{N}\right)$ is relatively compact. Therefore we have proved that $\left\{u_{n}\right\}_{n \geq 1} \subseteq C^{1}\left(T, \mathbb{R}^{N}\right)$ is relatively compact. We may assume that

$$
u_{n} \rightarrow u \text { in } C^{1}\left(T, \mathbb{R}^{N}\right) \text { as } n \rightarrow \infty .
$$

For every $\psi \in C_{0}^{1}\left((0, b), \mathbb{R}^{N}\right)$, we have

$$
\begin{aligned}
& \int_{0}^{b}\left(a\left(u_{n}^{\prime}(t)\right), \psi^{\prime}(t)\right)_{\mathbb{R}^{N}} d t+\int_{0}^{b}\left|u_{n}(t)\right|^{p-2}\left(u_{n}(t), \psi(t)\right)_{\mathbb{R}^{N}} d t \\
& +\int_{0}^{b}\left(A_{\lambda}\left(u_{n}(t)\right), \psi(t)\right)_{\mathbb{R}^{N}} d t=\int_{0}^{b}\left(g_{n}(t), \psi(t)\right)_{\mathbb{R}^{N}} d t, \text { for all } n \in \mathbb{N} .
\end{aligned}
$$

Passing to the limit as $n \rightarrow+\infty$, we obtain

$$
\begin{aligned}
& \int_{0}^{b}\left(a\left(u^{\prime}(t)\right), \psi^{\prime}(t)\right)_{\mathbb{R}^{N}} d t+\int_{0}^{b}|u(t)|^{p-2}(u(t), \psi(t))_{\mathbb{R}^{N}} d t \\
& +\int_{0}^{b}\left(A_{\lambda}(u(t)), \psi(t)\right)_{\mathbb{R}^{N}} d t=\int_{0}^{b}(g(t), \psi(t))_{\mathbb{R}^{N}} d t .
\end{aligned}
$$

Since $\psi \in C_{0}^{1}\left((0, b), \mathbb{R}^{N}\right)$ is arbitrary, it follows that

$$
\left\{\begin{array}{l}
-\left(a\left(u^{\prime}(t)\right)\right)^{\prime}+|u(t)|^{p-2} u(t)+A_{\lambda}(u(t))=g(t) \text { for a.a. } t \in T \\
u(0)=u(b)=0
\end{array}\right.
$$

hence $u=E_{\lambda}^{-1}(g)$. This proves Claim 3 .

Next, let $N_{3}: W_{0}^{1, p} \rightarrow 2^{L^{p^{\prime}}}$ be the multifunction defined by

$$
N_{3}(u)=S_{-F\left(\cdot, u(\cdot), u^{\prime}(.)\right)}^{p^{\prime}}+J(u) \text { for all } u \in W_{0}^{1, p} .
$$

Claim 4: $N_{3}$ is $\mathscr{P}_{w k c}\left(L^{p^{\prime}}\right)$-valued and is usc from $W_{0}^{1, p}$ into $L^{p^{\prime}}$ with the w-topology (denoted hereafter by $\left.L_{w}^{p^{\prime}}\right)$.

First we show the nonemptiness of the values of $N_{3}$. Hypotheses $\mathbf{H}(F)(i),(i i)$ do not imply that $F(t, x, y)$ is superpositionally measurable (see Hu-Papageorgiou [11], Example 7.2, p.227). So we cannot use directly in $F\left(\cdot, u(\cdot), u^{\prime}(\cdot)\right)$ the Yankov-von Neumann-Aumann selection theorem. To overcome this difficulty, we proceed as follows. Given $u \in W_{0}^{1, p}$ let $\left\{s_{n}\right\}_{n \geq 1},\left\{r_{n}\right\}_{n \geq 1}$ be two sequences of $\mathbb{R}^{N}$-valued step functions such that $s_{n}(t) \rightarrow u(t)$ and $r_{n}(t) \rightarrow u^{\prime}(t)$ for a.a. $t \in T$, as $n \rightarrow+\infty$ and $\left|s_{n}(t)\right| \leq|u(t)|$, $\left|r_{n}(t)\right| \leq\left|u^{\prime}(t)\right|$, a.e. on $T$ (note that $\left.u, u^{\prime} \in L^{p}\right)$. See Dinculeanu ([4] , p.99).

Then, for every $n \in \mathbb{N}$, hypothesis $\mathbf{H}(F)(i)$ implies that $t \rightarrow F\left(t, s_{n}(t), r_{n}(t)\right)$ is Lebesgue measurable. So, for this multifunction we can use the Yankov-von Neumann-Aumann selection theorem (see HuPapageorgiou [11], p.158) and for each $n \in \mathbb{N}$, we obtain $f_{n}: T \rightarrow \mathbb{R}^{N}$, measurable functions such that

$$
f_{n}(t) \in-F\left(t, s_{n}(t), r_{n}(t)\right) \text { for almost all } t \in T .
$$


On account of hypothesis $\mathbf{H}(F)(i i i)$, we see that $\left\{f_{n}\right\}_{n \geq 1} \subseteq L^{p^{\prime}}$ is bounded. So, we may assume that

$$
f_{n} \stackrel{w}{\longrightarrow} f \text { in } L^{p^{\prime}} \text { as } n \rightarrow+\infty \text {. }
$$

Invoking Proposition 3.9 of Hu-Papageorgiou ([11], p. 694), we obtain

$$
f(t) \in \overline{c o n v} \limsup _{n \rightarrow \infty}\left(-F\left(t, s_{n}(t), r_{n}(t)\right)\right) \subseteq-F\left(t, u(t), u^{\prime}(t)\right) \text { for a. a. } t \in T .
$$

The last inclusion follows from hypothesis $\mathbf{H}(F)(i i)$. So, we see that

$$
f \in S_{-F\left(\cdot, u(\cdot), u^{\prime}(\cdot)\right)}^{p^{\prime}}
$$

Therefore we have established the nonemptiness of the values of $N_{3}(\cdot)$.

It is clear that $N_{3}(\cdot)$ has bounded, closed, convex values. Hence, $N_{3}(u) \in \mathscr{P}_{w k c}\left(L^{p^{\prime}}\right)$ for all $u \in W_{0}^{1, p}$.

To show the upper semicontinuity of $N_{3}(\cdot)$, note that $N_{3}(\cdot)$ is bounded (that is maps bounded sets to bounded sets). Recall that bounded sets in $L^{p^{\prime}}$ with the relative weak topology are metrizable. So, according to Proposition 2.23 of Hu-Papageorgiou ([11], p. 43), to prove the claimed upper semicontinuity of $N_{3}(\cdot)$ it suffices to show that $G r N_{3} \subseteq W_{0}^{1, p} \times L_{w}^{p^{\prime}}$ is sequentially closed (see also Section 2). To this end consider $\left\{\left(u_{n}, g_{n}\right)\right\}_{n \geq 1} \subseteq \operatorname{GrN}_{3}$ such that

$$
u_{n} \rightarrow u \text { in } W_{0}^{1, p} \text { and } g_{n} \stackrel{w}{\longrightarrow} g \text { in } L^{p^{\prime}} \text { as } n \rightarrow+\infty .
$$

Using the compact embedding of $W_{0}^{1, p}$ into $C\left(T, \mathbb{R}^{N}\right)$, we have

$$
u_{n}(t) \rightarrow u(t) \text { for a.a. } t \in T, \text { as } n \rightarrow \infty .
$$

Also, we can say that

$$
u_{n}^{\prime}(t) \rightarrow u^{\prime}(t) \text { for a.a. } t \in T, \text { as } n \rightarrow \infty
$$

Then, as before, we have

$$
\begin{aligned}
g(t) & \in \overline{c o n v} \limsup _{n \rightarrow \infty}\left(-F\left(t, u_{n}(t), u_{n}^{\prime}(t)\right)+\left|u_{n}(t)\right|^{p-2} u_{n}(t)\right) \\
& \subseteq-F\left(t, u(t), u^{\prime}(t)\right)+|u(t)|^{p-2} u(t) \text { for a. a. } t \in T,
\end{aligned}
$$

hence $g \in N_{3}(u)$. This proves Claim 4 .

We next consider the set

$$
S=\left\{u \in W_{0}^{1, p}: u \in \beta E_{\lambda}^{-1} N_{3}(u), 0<\beta<1\right\} .
$$

Claim 5: $S \subseteq W_{0}^{1, p}$ is bounded.

Let $u \in S$. We have

$$
E_{\lambda}\left(\frac{1}{\beta} u\right) \in N_{3}(u)
$$

hence

$$
\left\{\begin{array}{c}
-\left(a\left(\frac{1}{\beta} u^{\prime}(t)\right)\right)^{\prime}+\frac{1}{\beta}|u(t)|^{p-2} u(t)+A_{\lambda}\left(\frac{1}{\beta} u(t)\right) \\
=f(t)+|u(t)|^{p-2} u(t) \text { for a.a. } t \in T \\
u(0)=u(b)=0, f \in S_{-F\left(\cdot, u(\cdot), u^{\prime}(\cdot)\right)}^{p}
\end{array}\right.
$$

Hypothesis $\mathbf{H}(F)(i v)$ implies that given $\varepsilon>0$, we can find $M_{1}=M_{1}(\varepsilon)>0$ such that for a.a. $t \in T$, all $|x| \geq M_{1}$, all $y \in \mathbb{R}^{N}$ and all $v \in-F(t, x, y)$, we have

$$
(v, x)_{\mathbb{R}^{N}} \leq[\boldsymbol{\theta}(t)+\boldsymbol{\varepsilon}]|x|^{p} .
$$


From hypothesis $\mathbf{H}(F)(i i i)$ we see that for a. a. $t \in T$, all $|x|<M_{1}$, all $y \in \mathbb{R}^{N}$ and all $v \in-F(t, x, y)$, we have

$$
|v| \leq \eta_{1, M_{1}}(t)+\eta_{2, M_{1}}(t)|y|^{p-1}
$$

therefore

$$
(v, x)_{\mathbb{R}^{N}} \leq M_{1} \eta_{1, M_{1}}(t)+M_{1} \eta_{2, M_{1}}(t)|y|^{p-1} .
$$

Consequently, finally, for a. a. $t \in T$, all $x, y \in \mathbb{R}^{N}$ and all $v \in-F(t, x, y)$ we have

$$
(v, x)_{\mathbb{R}^{N}} \leq(\theta(t)+\varepsilon)|x|^{p}+C_{8}|y|^{p-1}+\mu(t)
$$

for some $C_{8}>0$ and $\mu \in L^{1}(T)_{+}$. Returning to (3.12), taking the inner product with $u(t)$, integrating over $T=[0, b]$, performing an integration by parts and using (3.13), we obtain

$$
\frac{C_{0}}{\beta^{p-1}}\left\|u^{\prime}\right\|_{p}^{p}+\left(\frac{1}{\beta^{p-1}}-1\right)\|u\|_{p}^{p} \leq \int_{0}^{b}(\theta(t)+\varepsilon)|u|^{p} d t+C_{8} \int_{0}^{b}\left|u^{\prime}\right|^{p-1} d t+\|\mu\|_{1} .
$$

Employing (2.2) and Holder's inequality, we arrive at

$$
C_{0}\left\|u^{\prime}\right\|_{p}^{p}-\int_{0}^{b} \theta(t)|u|^{p} d t-\frac{\varepsilon}{\widehat{\lambda}_{1}}\left\|u^{\prime}\right\|_{p}^{p} \leq C_{9}\left\|u^{\prime}\right\|_{p}^{p-1}+\|\mu\|_{1}
$$

for some $C_{9}>0$. Therefore

$$
\left(C_{10}-\frac{\varepsilon}{\widehat{\lambda}_{1}}\right)\left\|u^{\prime}\right\|_{p}^{p} \leq C_{9}\left\|u^{\prime}\right\|_{p}^{p-1}+\|\mu\|_{1} \text { for some } C_{10}>0
$$

(see hypothesis $\mathbf{H}(F)(i v)$ and Proposition 2.3). Choosing $\varepsilon \in\left(0, C_{10} \widehat{\lambda}_{1}\right)$, from (3.14) we have

$$
\left\|u^{\prime}\right\|_{p}^{p} \leq C_{11}\left(\left\|u^{\prime}\right\|_{p}^{p-1}+1\right) \text { for some } C_{11}>0 .
$$

From (3.15) and Poincare's inequality it follows that

$$
\|u\| \leq C_{12} \text { for some } C_{12}>0, \text { all } u \in S,
$$

and we conclude that $S \subseteq W_{0}^{1, p}$ is bounded. This proves Claim 5 .

Now Claims 3, 4, and 5 permit the use of Proposition 2.2. So, we can find $u_{\lambda} \in D(V)$ such that

$$
u_{\lambda} \in E_{\lambda}^{-1} N_{3}\left(u_{\lambda}\right) .
$$

Evidently $u_{\lambda} \in C^{1}\left(T, \mathbb{R}^{N}\right)$ and it is a solution of problem (3.9).

Eventually we will let $\lambda \rightarrow 0^{+}$, to produce a solution of problem (1.1). To do this, we need an additional auxiliary result.

Let $1<r, r^{\prime}<\infty, \frac{1}{r}+\frac{1}{r^{\prime}}=1$ and let $\mathscr{A}: L^{r} \rightarrow 2^{L^{r^{\prime}}}$ be the lifting (realization) of $A(\cdot)$ defined by

$$
\mathscr{A}(u)=\left\{h \in L^{r^{\prime}}: h(t) \in A(u(t)) \text { for a.a. } t \in T\right\}
$$

for all $u \in D(\mathscr{A})$, where

$$
D(\mathscr{A})=\left\{y \in L^{r}: y(t) \in D(A) \text { for a.a. } t \in T, S_{A(u(\cdot))}^{r^{\prime}} \neq \varnothing\right\} .
$$

Since $0 \in D(\mathscr{A})$, it follows that $D(\mathscr{A}) \neq \varnothing$. 
The next Proposition is known for Hilbert spaces (see Brezis [3], p.25). However, our proof here is different and can be easily extended to Banach spaces.

Proposition 3.4. If hypotheses $\mathbf{H}(a)$ hold, then $\mathscr{A}: L^{r} \rightarrow 2^{L^{r^{\prime}}}$ is maximal monotone.

Proof. Let $e: \mathbb{R}^{N} \rightarrow \mathbb{R}^{N}$ be defined by $e(x)=|x|^{r-2} x$. This map is continuous and strictly monotone (hence maximal monotone). The Nemitsky operator corresponding to $e(\cdot)$ is the map $J: L^{r} \rightarrow L^{r^{\prime}}$ defined by

$$
J(u)(\cdot)=|u(\cdot)|^{r-2} u(\cdot) \text { for all } u \in L^{r} .
$$

From the proof of Proposition 3.2, we know that to prove the maximality of $\mathscr{A}(\cdot)$ it suffices to show that

$$
R(\mathscr{A}+J)=L^{r^{\prime}} .
$$

We know that $x \rightarrow(A+e)(x)$ is maximal monotone and coercive (recall that $0 \in A(0)$ ). Therefore $R(A+e)=\mathbb{R}^{N}$ (see Gasinski-Papageorgiou [8], Corollary 3.2.31, p. 319).

Let $h \in L^{r^{\prime}}$ and consider the multifunction $L: T \rightarrow 2^{\mathbb{R}^{N}}$ defined by

$$
L(t)=\left\{x \in \mathbb{R}^{N}: A(x)+e(x) \ni h(t)\right\} .
$$

From the surjectivity of $(A+e)(\cdot)$, we see that $L(t) \neq \varnothing$ for a.a. $t \in T$. Consider the function $\eta$ : $T \times \mathbb{R}^{N} \rightarrow \mathbb{R}^{N} \times \mathbb{R}^{N}$ defined by

$$
\eta(t, x)=(x, h(t)-e(x)) .
$$

Then $\eta$ is a Carathéodory function (that is, for all $x \in \mathbb{R}^{N}, t \rightarrow \eta(t, x)$ is measurable and for almost all $t \in T, x \rightarrow \eta(t, x)$ is continuous). Therefore $\eta(\cdot, \cdot)$ is jointly measurable (see Hu-Papageorgiou [11], Proposition 1.6, p. 142). We have

$$
G r L=\left\{(t, x) \in T \times \mathbb{R}^{N}: \eta(t, x) \in G r A\right\}=\eta^{-1}(G r A) .
$$

Recall that $G r A \subseteq \mathbb{R}^{N} \times \mathbb{R}^{N}$ is closed (since $A$ is maximal monotone). Then the joint measurability of $\eta$ implies that

$$
G r L=\eta^{-1}(G r A) \in \mathscr{L}_{T} \times \mathscr{B}\left(\mathbb{R}^{N}\right),
$$

where $\mathscr{L}_{T}$ is the Lebesgue $\sigma$-field of $T$ and $\mathscr{B}\left(\mathbb{R}^{N}\right)$ is the Borel $\sigma$-field of $\mathbb{R}^{N}$. We can apply Yankovvon Neumann-Aumann selection theorem and produce a measurable map $u: T \rightarrow \mathbb{R}^{N}$ such that $u(t) \in$ $L(t)$ for a.a. $t \in T$. Then we have

$$
h(t) \in A(u(t))+e(u(t)) \text { for a.a. } t \in T .
$$

We take the inner product with $u(t) \in \mathbb{R}^{N}$ and obtain

$$
|u(t)|^{r} \leq|h(t)||u(t)| \text { for a.a. } t \in T
$$

(recall that $0 \in A(0)$ ), hence

$$
|u(t)|^{r-1} \leq|h(t)| \text { for a.a. } t \in T,
$$

and it follows that $u \in L^{r}$. Therefore, finally we have $\mathscr{A}(u)+J(u) \ni h$ with $h \in L^{r^{\prime}}$, and so, (3.16) holds, implying that $\mathscr{A}(\cdot)$ is maximal monotone.

Remark 3.1. In fact the result is true if $\mathbb{R}^{N}$ is replaced by a reflexive Banach space $\mathrm{X}$. The same proof with minor changes works in this more general case. 


\section{EXISTENCE THEOREMS}

In this section we prove several existence theorems for problem (1.1). First we prove an existence theorem under slightly stronger hypotheses on the multivalued perturbation $F(t, x, y)$ ("convex" problem, since $F$ is convex valued). Specifically, we assume:

$\mathbf{H}(F)_{1}: F: T \times \mathbb{R}^{N} \times \mathbb{R}^{N} \rightarrow \mathscr{P}_{k c}\left(\mathbb{R}^{N}\right)$ satisfies $\mathbf{H}(F)(i),(i i),(i v)$ as well as $\mathbf{H}(F)(i i i)$ where the exponent $p-1$ is replaced by $q=\max \left\{1, \frac{p-1}{2}\right\}$ and $\eta_{1, k} \in L^{2}(T)_{+}$.

Theorem 4.1. If hypotheses $\mathbf{H}(a), \mathbf{H}(A), \mathbf{H}(F)_{1}$ hold, then problem (1.1) has a solution $u \in C^{1}\left(T, \mathbb{R}^{N}\right)$.

Proof. Let $\lambda_{n} \rightarrow 0^{+}$and let $u_{n}=u_{\lambda_{n}} \in C^{1}\left(T, \mathbb{R}^{N}\right)$ be a solution of (3.9) with $\lambda=\lambda_{n}, n \in \mathbb{N}$ (see Proposition 3.3). We have

$$
\left\{\begin{array}{l}
-\left(a\left(u_{n}^{\prime}(t)\right)\right)^{\prime}=A_{\lambda_{n}}\left(u_{n}(t)\right)+f_{n}(t) \text { for a.a. } t \in T \\
u_{n}(0)=u_{n}(b)=0
\end{array}\right.
$$

with $f_{n} \in S_{F\left(\cdot, u_{n}(\cdot), u_{n}^{\prime}(\cdot)\right)}^{p^{\prime}}$ for all $n \in \mathbb{N}$. As before, acting on (4.1) with $u_{n}(t)$ we obtain

$$
C_{0}\left\|u_{n}^{\prime}\right\|_{p}^{p} \leq \int_{0}^{b}\left(-f_{n}(t), u_{n}(t)\right)_{\mathbb{R}^{N}} d t \text { for all } n \in \mathbb{N}
$$

From (3.13), we have

$$
\left(-f_{n}(t), u_{n}(t)\right)_{\mathbb{R}^{N}} \leq[\theta(t)+\varepsilon]\left|u_{n}(t)\right|^{p}+C_{8}\left|u_{n}^{\prime}(t)\right|^{p-1}+\mu(t) \text { for a.a. } t \in T .
$$

Using this in (4.2), as in the proof of Proposition 3.3, and invoking Proposition 2.3, we deduce that

$$
\left\{u_{n}\right\}_{n \geq 1} \subseteq W_{0}^{1, p} \text { is bounded. }
$$

So, we may assume that

$$
u_{n} \stackrel{w}{\longrightarrow} u \text { in } W_{0}^{1, p} \text { and } u_{n} \rightarrow u \text { in } C\left(T, \mathbb{R}^{N}\right)
$$

We return to (4.1) and take the inner product with $A_{\lambda_{n}} u_{n}(t)$. Integrating over $T=[0, b]$, we have

$$
\begin{aligned}
& \int_{0}^{b}\left(-\left(a\left(u_{n}^{\prime}(t)\right)\right)^{\prime}, A_{\lambda_{n}}\left(u_{n}(t)\right)\right)_{\mathbb{R}^{N}} d t+\left\|A_{\lambda_{n}}\left(u_{n}\right)\right\|_{2}^{2} \\
& \leq \int_{0}^{b}\left|f_{n}(t)\right|\left|A_{\lambda_{n}}\left(u_{n}(t)\right)\right| d t .
\end{aligned}
$$


Note that $A_{\lambda_{n}}\left(u_{n}\right)=\mathscr{A}_{\lambda_{n}}\left(u_{n}\right) \in C\left(T, \mathbb{R}^{N}\right)$. Performing an integration by parts in the first integral, we have

$$
\begin{aligned}
& \int_{0}^{b}\left(-\left(a\left(u_{n}^{\prime}(t)\right)\right)^{\prime}, A_{\lambda_{n}}\left(u_{n}(t)\right)\right)_{\mathbb{R}^{N}} d t \\
& =-\left(\left(a\left(u_{n}^{\prime}(b)\right)\right)^{\prime}, A_{\lambda_{n}}\left(u_{n}(b)\right)\right)_{\mathbb{R}^{N}}+\left(\left(a\left(u_{n}^{\prime}(0)\right)\right)^{\prime}, A_{\lambda_{n}}\left(u_{n}(0)\right)\right)_{\mathbb{R}^{N}} \\
& +\int_{0}^{b}\left(a\left(u_{n}^{\prime}(t)\right), \frac{d}{d t} A_{\lambda_{n}}\left(u_{n}(t)\right)\right)_{\mathbb{R}^{N}} d t \\
& =\int_{0}^{b}\left(a\left(u_{n}^{\prime}(t)\right), \frac{d}{d t} A_{\lambda_{n}}\left(u_{n}(t)\right)\right)_{\mathbb{R}^{N}} d t
\end{aligned}
$$

(since $u_{n}(b)=u_{n}(0)=0$ and $A_{\lambda_{n}}(0)=0$ ).

Recall that for $n \in \mathbb{N}, A_{\lambda_{n}}: \mathbb{R}^{N} \rightarrow \mathbb{R}^{N}$ is Lipschitz continuous (see Proposition 2.1). So, by the Rademacher's theorem (see, for example Gasinski-Papageorgiou [8], Theorem 1.5.8, p. 56), we know that $A_{\lambda_{n}}(\cdot)$ is differentiable at every $x \in \mathbb{R}^{N} \backslash S_{1}$ with $\left|S_{1}\right|_{N}=0$ (here $|\cdot|_{N}$ denotes the Lebesgue measure on $\left.\mathbb{R}^{N}\right)$. Then, for all $x \in \mathbb{R}^{N} \backslash S_{1}$, all $h \in \mathbb{R}^{N}$ and all $\tau>0$, we have

$$
\left(h, \frac{A_{\lambda_{n}}(x+\tau h)-A_{\lambda_{n}}(x)}{\tau}\right)_{\mathbb{R}^{N}} \geq 0 \text { (since } A_{\lambda_{n}} \text { is monotone), }
$$

hence sending $\tau \rightarrow 0^{+}$we get

$$
\left(h, A_{\lambda_{n}}^{\prime}(x) h\right)_{\mathbb{R}^{N}} \geq 0 .
$$

From the chain rule of Marcus-Mizel [14], we have

$$
\frac{d}{d t} A_{\lambda_{n}}\left(u_{n}(t)\right)=A_{\lambda_{n}}^{\prime}\left(u_{n}(t)\right) u_{n}^{\prime}(t) \text { for a.a. } t \in T .
$$

If $a(x)=k(x) x(\mathrm{cf} . \mathbf{H}(a))$, then

$$
\begin{aligned}
& \int_{0}^{b}\left(-\left(a\left(u_{n}^{\prime}(t)\right)\right)^{\prime}, A_{\lambda_{n}}\left(u_{n}(t)\right)\right)_{\mathbb{R}^{N}} d t \\
& =\int_{0}^{b}\left(a\left(u_{n}^{\prime}(t)\right), \frac{d}{d t} A_{\lambda_{n}}\left(u_{n}(t)\right)\right)_{\mathbb{R}^{N}} d t \\
& =\int_{0}^{b} k\left(u_{n}^{\prime}(t)\right)\left(u_{n}^{\prime}(t), A_{\lambda_{n}}^{\prime}\left(u_{n}(t)\right) u_{n}^{\prime}(t)\right)_{\mathbb{R}^{N}} d t \\
& \geq 0(\text { see }(4.6) \text { and }(4.5))
\end{aligned}
$$

Similarly if $a(x)=\left(k_{m}(x) x_{m}\right)_{m=1}^{N}$.

Let $M_{1}=\sup \left\|u_{n}\right\|_{C\left(T, \mathbb{R}^{N}\right)}$. Then hypothesis $\mathbf{H}(F)_{1}(i i i)$ implies that

$$
\left|f_{n}(t)\right| \leq \eta_{1, M_{1}}(t)+\eta_{2, M_{1}}(t)\left|u_{n}^{\prime}(t)\right|^{q} \text { for a.a. } t \in T, \text { all } n \in \mathbb{N}
$$

with $\eta_{1, M_{1}} \in L^{2}(T), \eta_{2, M_{1}} \in L^{\infty}(T)$. Since $u_{n}^{\prime} \in C\left(T, \mathbb{R}^{N}\right)$ (recall that $u_{n} \in D(V)$ for all $n \in \mathbb{N}$ ) we see that $f_{n} \in L^{2}\left(T, \mathbb{R}^{N}\right)$ for all $n \in \mathbb{N}$. Moreover, by (4.3) and (4.8) it follows that $\left\{f_{n}\right\}_{n \geq 1}$ is bounded in 
$L^{2}\left(T, \mathbb{R}^{N}\right)$. Then

$$
\int_{0}^{b}\left|f_{n}(t)\right|\left|A_{\lambda_{n}}\left(u_{n}(t)\right)\right| d t \leq\left\|f_{n}\right\|_{2}\left\|\mathscr{A}_{\lambda_{n}}\left(u_{n}\right)\right\|_{2} \leq M_{2}\left\|\mathscr{A}_{\lambda_{n}}\left(u_{n}\right)\right\|_{2}
$$

for some $M_{2}>0$, all $n \in \mathbb{N}$. Returning to (4.4) and using (4.7) and (4.9), we have

$$
\left\|\mathscr{A}_{\lambda_{n}}\left(u_{n}\right)\right\|_{2}^{2} \leq M_{2}\left\|\mathscr{A}_{\lambda_{n}}\left(u_{n}\right)\right\|_{2},
$$

hence

$$
\left\{\mathscr{A}_{\lambda_{n}}\left(u_{n}\right)\right\}_{n \geq 1} \subseteq L^{2} \subseteq L^{p^{\prime}} \text { is bounded (recall that } p^{\prime} \leq 2 \leq p \text { ). }
$$

So, we may assume that

$$
\mathscr{A}_{\lambda_{n}}\left(u_{n}\right) \stackrel{w}{\longrightarrow} g \text { in } L^{2} \text { and } L^{p^{\prime}} \text { as } n \rightarrow \infty .
$$

As in the proof of Proposition 3.3 (see Claim 3), we obtain that $\left\{u_{n}\right\}_{n \geq 1} \subseteq C^{1}\left(T, \mathbb{R}^{N}\right)$ is relatively compact. We may assume that

$$
u_{n} \rightarrow u \text { in } C^{1}\left(T, \mathbb{R}^{N}\right) \text { and so } a\left(u_{n}^{\prime}(t)\right) \rightarrow a(u(t)) \text { for all } t \in T .
$$

Also, we have

$$
f_{n} \stackrel{w}{\longrightarrow} f \text { in } L^{p^{\prime}}\left(T, \mathbb{R}^{N}\right) \text { with } f \in S_{F\left(\cdot, u(\cdot), u^{\prime}(.)\right)}^{p^{\prime}}
$$

For every $\psi \in C_{0}^{1}\left((0, b), \mathbb{R}^{N}\right)$, we have

$$
\int_{0}^{b}\left(-a\left(u_{n}^{\prime}(t)\right), \psi^{\prime}(t)\right)_{\mathbb{R}^{N}} d t=\int_{0}^{b}\left(A_{\lambda_{n}}\left(u_{n}(t)\right), \psi(t)\right)_{\mathbb{R}^{N}} d t+\int_{0}^{b}\left(f_{n}(t), \psi(t)\right)_{\mathbb{R}^{N}} d t
$$

for all $n \in \mathbb{N}$. Passing to the limit as $n \rightarrow \infty$, we obtain

$$
\int_{0}^{b}\left(-a\left(u^{\prime}(t)\right)^{\prime}, \psi^{\prime}(t)\right)_{\mathbb{R}^{N}} d t=\int_{0}^{b}(g(t), \psi(t))_{\mathbb{R}^{N}} d t+\int_{0}^{b}(f(t), \psi(t))_{\mathbb{R}^{N}} d t
$$

(see (4.10) and (4.11). Since $\psi \in C_{0}^{1}\left((0, b), \mathbb{R}^{N}\right)$ is arbitrary, it follows that

$$
\left(a\left(u^{\prime}(t)\right)\right)^{\prime}=g(t)+f(t) \text { for a.a. } t \in T, u(0)=u(b)=0,
$$

with $f \in S_{F\left(\cdot, u(\cdot), u^{\prime}(.)\right)}^{p^{\prime}}$ (see (4.12)). Clearly $u(\cdot)$ will be a solution of $(1.1)$ if we show that $g \in \mathscr{A}(u)$. This follows easily from (4.3), (4.10), Proposition 2.1 and Proposition 3.4 (applied to the special case $r=2)$.

We also prove an existence theorem for problem (1.1) in the case where $F$ is nonconvex valued ("nonconvex" problem). Now the hypotheses on the multivalued perturbation $F$ are:

$\mathbf{H}(F)_{2}: F: T \times \mathbb{R}^{N} \times \mathbb{R}^{N} \rightarrow \mathscr{P}_{k}\left(\mathbb{R}^{N}\right)$ is a multifunction such that

(i) $(t, x, y) \rightarrow F(t, x, y)$ is graph measurable;

(ii) for a. a. $t \in T,(x, y) \rightarrow F(t, x, y)$ is lsc;

(iii) same as hypothesis $\mathbf{H}(F)_{1}($ iii $)$;

(iv) same as hypothesis $\mathbf{H}(F)(i v)$.

Theorem 4.2. If hypotheses $\mathbf{H}(a), \mathbf{H}(A), \mathbf{H}(F)_{2}$ hold, then problem (1.1) has a solution $u \in C^{1}\left(T, \mathbb{R}^{N}\right)$. 
Proof. Let $N: W_{0}^{1, p} \rightarrow \mathscr{P}_{f}\left(L^{p^{\prime}}\right)$ be the multifunction defined by

$$
N(u)=S_{F\left(\cdot, u(\cdot), u^{\prime}(.)\right)}^{p^{\prime}} \text { for all } u \in W_{0}^{1, p} .
$$

We claim that $N(\cdot)$ is 1sc. According to Proposition 2.26 of Hu-Papageorgiou ([11], p.45) it suffices to show that for every $v \in L^{p^{\prime}}$, the function $u \rightarrow d_{p^{\prime}}(v, N(u))=\inf \left\{\|v-f\|_{p^{\prime}}: f \in N(u)\right\}$ is upper semicontinuous. From Theorem 3.24 of Hu-Papageorgiou ([11], p.183), we have

$$
d_{p^{\prime}}(v, N(u))=\left(\int_{0}^{b}\left[d\left(v(t), F\left(t, u(t), u^{\prime}(t)\right)\right)\right]^{p^{\prime}} d t\right)^{\frac{1}{p^{\prime}}} .
$$

We need to show that for every $\xi>0$, the superlevel set

$$
U_{\xi}=\left\{u \in W_{0}^{1, p}: d_{p^{\prime}}(v, N(u)) \geq \xi\right\}
$$

is closed. So, suppose that $\left\{u_{n}\right\}_{n \geq 1} \subseteq U_{\xi}$ and assume that $u_{n} \rightarrow u$ in $W_{0}^{1, p}$. Using (4.13) we have

$$
\int_{0}^{b}\left[d\left(v(t), F\left(t, u_{n}(t), u_{n}^{\prime}(t)\right)\right)\right]^{p^{\prime}} d t \geq \xi^{p^{\prime}} \text { for all } n \in \mathbb{N} .
$$

We may assume that

$$
u_{n}(t) \rightarrow u(t) \text { for all } t \in T \text { and } u_{n}^{\prime}(t) \rightarrow u^{\prime}(t) \text { for a. a. } t \in T .
$$

From Fatou's lemma, we have

$$
\int_{0}^{b} \limsup _{n \rightarrow \infty}\left[d\left(v(t), F\left(t, u_{n}(t), u_{n}^{\prime}(t)\right)\right)\right]^{p^{\prime}} d t \geq \xi^{p^{\prime}} .
$$

Hypothesis $\mathbf{H}(F)_{2}(i i)$ implies that $(x, y) \rightarrow d_{p^{\prime}}(v(t), F(t, x, y))$ is upper semicontinuous. So,

$$
\int_{0}^{b} \limsup _{n \rightarrow \infty}\left[d\left(v(t), F\left(t, u(t), u^{\prime}(t)\right)\right)\right]^{p^{\prime}} d t \geq \xi^{p^{\prime}}
$$

(see (4.14)) hence

$$
d_{p^{\prime}}(v, N(u)) \geq \xi
$$

that is, $u \in U_{\xi}$, and $N(\cdot)$ is lsc. Also $N(\cdot)$ has decomposable values. Therefore we can apply Theorem 8.7 of Hu-Papageorgiou ([11], p.245) and obtain a continuous map $d: W_{0}^{1, p} \rightarrow L^{p^{\prime}}$ such that

$$
d(u) \in N(u) \text { for all } u \in W_{0}^{1, p} .
$$

Then, as in the proof of Proposition 3.3, we show that for every $\lambda>0$ the problem

$$
\left(a\left(u^{\prime}(t)\right)\right)^{\prime}=A_{\lambda}(u(t))+d(u)(t) \text { for a.a. } t \in T, u(0)=u(b)=0
$$

admits a solution $u_{\lambda} \in C^{1}\left(T, \mathbb{R}^{N}\right)$. Passing to the limit as $\lambda \rightarrow 0^{+}$and reasoning as in the proof of Theorem 4.1, we produce a solution $u \in C^{1}\left(T, \mathbb{R}^{N}\right)$ for the nonconvex problem.

We can relax the condition on the map $a(\cdot)$ at the expense of strengthening the condition on $A(\cdot)$. The new hypotheses on $a(\cdot)$ and $A(\cdot)$ are: 
$\mathbf{H}(a)^{\prime}: a: \mathbb{R}^{N} \rightarrow \mathbb{R}^{N}$ is a continuous map such that

(i) $a(\cdot)$ is strictly monotone;

(ii) $(a(x), x)_{\mathbb{R}^{N}} \geq C_{0}|x|^{p}$ for all $x \in \mathbb{R}^{N}$, some $C_{0}>0$, and with $p \geq 2$.

$\mathbf{H}(A)^{\prime}: A: \mathbb{R}^{N} \rightarrow 2^{\mathbb{R}^{N}}$ is a maximal monotone map such that $D(A)=\mathbb{R}^{N}$ and $0 \in A(0)$.

Remark 4.1. The hypothesis that $D(A)=\mathbb{R}^{N}$ precludes from considerations maps of the form $A=\partial i_{C}$ where $i_{C}$ is the indicator function of a set $C \in \mathscr{P}_{f c}\left(\mathbb{R}^{N}\right)$. Such maps arise in problems with unilateral constraints (variational inequalities).

Theorem 4.3. If hypotheses $\mathbf{H}(a)^{\prime}, \mathbf{H}(A)^{\prime}, \mathbf{H}(F)$ hold, then problem (1.1) has a solution $u \in C^{1}\left(T, \mathbb{R}^{N}\right)$.

Proof. The particular structure of $a(\cdot)$ considered in hypothesis $\mathbf{H}(a)$ (namely that $a(x)=k(x) x$ or that $a(x)=\left(k_{m}(x) x_{m}\right)_{m=1}^{N}$ was only used in the proof of Theorem 4.1 in order to show the $L^{2}$-boundedness of $\left\{\mathscr{A}_{\lambda_{n}}\left(u_{n}\right)\right\}_{n \geq 1}$. So, Propositions 3.1, 3.2, 3.3 are still valid in the present setting. Moreover, if $\left\{u_{n}\right\}_{n \geq 1} \subseteq C^{1}\left(T, \mathbb{R}^{N}\right)$ are as in the proof of Theorem 4.1, from the first part of that proof we know that $\left\{u_{n}\right\}_{n \geq 1} \subseteq W_{0}^{1, p}$ is bounded, hence $\left\{u_{n}\right\}_{n \geq 1} \subseteq C\left(T, \mathbb{R}^{N}\right)$ is relatively compact (recall that $W_{0}^{1, p} \hookrightarrow$ $C\left(T, \mathbb{R}^{N}\right)$ compactly). Therefore, we can find $M_{3}>0$ such that

$$
\left\|u_{n}\right\|_{C\left(T, \mathbb{R}^{N}\right)} \leq M_{3} \text { for all } n \in \mathbb{N} .
$$

Since $J_{\lambda_{n}}(0)=0$ and $J_{\lambda_{n}}(\cdot)$ is nonexpansive (see Proposition 2.1), we have

$$
\left\|J_{\lambda_{n}}\left(u_{n}(\cdot)\right)\right\|_{C\left(T, \mathbb{R}^{N}\right)} \leq M_{3} \text { for all } n \in \mathbb{N}
$$

Then we have

$$
A_{\lambda_{n}}\left(u_{n}(t)\right) \in A\left(J_{\lambda_{n}}\left(u_{n}(t)\right)\right) \subseteq A\left(\bar{B}_{M_{3}}(0)\right),
$$

where $\bar{B}_{M_{3}}(0)=\left\{v \in \mathbb{R}^{N}:|v| \leq M_{3}\right\}$. But, from Theorem 1.28 of Hu-Papageorgiou ([11], p. 308) we know that $A(\cdot)$ is usc with $\mathscr{P}_{k c}\left(\mathbb{R}^{N}\right)$-values. So, by Corollary 2.20 of Hu-Papageorgiou ([11], p. 42) we have that $A\left(\bar{B}_{M_{3}}(0)\right) \in \mathscr{P}_{k}\left(\mathbb{R}^{N}\right)$. Hence

$$
\left|A_{\lambda_{n}}\left(u_{n}(t)\right)\right| \leq M_{4} \text { for some } M_{4}>0, \text { all } n \in \mathbb{N} \text {, all } t \in T
$$

(see (4.15)). Now the rest of the proof proceeds as the corresponding part of the proof of Theorem 4.1.

We can derive a "nonconvex" version of this existence theorem, as well. The hypotheses on the multivalued perturbation are the following:

$\mathbf{H}(F)_{2}^{\prime}: F: T \times \mathbb{R}^{N} \times \mathbb{R}^{N} \rightarrow \mathscr{P}_{k}\left(\mathbb{R}^{N}\right)$ is a multifunction satisfying $\mathbf{H}(F)_{2}(i),(i i)$ and $\mathbf{H}(F)(i i i),(i v)$.

Theorem 4.4. If hypotheses $\mathbf{H}(a)^{\prime}, \mathbf{H}(A)^{\prime}, \mathbf{H}(F)_{2}^{\prime}$ hold, then problem (1.1) has a solution $u \in C^{1}\left(T, \mathbb{R}^{N}\right)$.

Proof. We just combine the proofs of Theorems 4.2 and 4.3. 


\section{AN EXAMPLE}

In this section, we present an example of a differential variational inequality (a problem with unilateral constraints) to which our general existence theory applies.

Let $\mathbb{R}_{+}^{N}$ be the usual positive cone of $\mathbb{R}^{N}$ (that is, $\mathbb{R}_{+}^{N}=\left\{x=\left(x_{m}\right)_{m=1}^{N} \in \mathbb{R}^{N}: x_{m} \geq 0\right\}$. This is a closed, convex cone. Let $i_{\mathbb{R}_{+}^{N}}(\cdot)$ be the indicator function of $\mathbb{R}_{+}^{N}$, that is,

$$
i_{\mathbb{R}_{+}^{N}}(x)=\left\{\begin{array}{ccc}
0 & \text { if } & x \in \mathbb{R}_{+}^{N}, \\
+\infty & \text { if } & x \notin \mathbb{R}_{+}^{N} .
\end{array}\right.
$$

It is easily seen that $i_{\mathbb{R}_{+}^{N}}(\cdot) \in \Gamma_{0}\left(\mathbb{R}^{N}\right)$, the space of all proper, convex, lower semicontinuous functions on $\mathbb{R}^{N}$ (See Gasinski-Papageorgiou [8], Definition 4.21, p. 488). We set

$$
A(x)=\partial i_{\mathbb{R}_{+}^{N}}(x)=N_{\mathbb{R}_{+}^{N}}(x)=\text { the normal cone to } \mathbb{R}_{+}^{N} \text { at } x,
$$

where " $\partial "$ "stands for the subdifferential in the sense of convex analysis. We know that for $x=\left(x_{m}\right)_{m=1}^{N} \in$ $\mathbb{R}_{+}^{N}$ we have

$$
N_{\mathbb{R}_{+}^{N}}(x)=\left\{\begin{array}{ccc}
0 & \text { if } & x_{m}>0 \text { for all } m=1, \ldots, N, \\
-\mathbb{R}_{+}^{N} \cap\{x\}^{\perp} & \text { if } & x_{m}=0 \text { for at least one } m=1, \ldots, N .
\end{array}\right.
$$

So, $D(A)=\mathbb{R}_{+}^{N}$. Then problem (1.1) reduces to the following multivalued differential variational inequality

$$
\left\{\begin{array}{c}
\left(a\left(u^{\prime}(t)\right)\right)^{\prime} \in F\left(t, u(t), u^{\prime}(t)\right) \text { a.e. on } \\
\left\{t \in T: u_{m}(t)>0 \text { for all } m=1, \ldots, N\right\} \\
\left(a\left(u^{\prime}(t)\right)\right)^{\prime} \in F\left(t, u(t), u^{\prime}(t)\right)-h(t) \text { a.e. on } \\
\left\{t \in T: u_{m}(t)=0 \text { for some } m=1, \ldots, N\right\} \\
h(t) \in \mathbb{R}_{+}^{N},(h(t), u(t))_{\mathbb{R}^{N}}=0 \text { for a.a. } t \in T, \\
u=\left(u_{m}\right)_{m=1}^{N} \in C^{1}\left(T, \mathbb{R}^{N}\right) \\
u_{m}(t) \geq 0 \text { for all } t \in T, \text { all } m=1, \ldots, N, \\
u(0)=u(b)=0 .
\end{array}\right.
$$

If $F$ satisfies hypotheses $\mathbf{H}(F)$ or $\mathbf{H}(F)_{2}$, then (5.1) admits a solution (see Theorems 4.1, 4.2).

If $F(t, x, y)=f(t, x, y)$ is single-valued, then (5.1) has the following more familiar form (recall that on $\left.\mathbb{R}^{N}, x \leq y \Longleftrightarrow y-x \in \mathbb{R}_{+}^{N}\right)$ :

$$
\left\{\begin{array}{l}
\left(a\left(u^{\prime}(t)\right)\right)^{\prime}=f\left(t, u(t), u^{\prime}(t)\right) \text { a.e. } \\
\text { on }\left\{t \in T: u_{m}(t)>0 \text { for all } m=1, \ldots, N\right\} \\
\left(a\left(u^{\prime}(t)\right)\right)^{\prime} \leq f\left(t, u(t), u^{\prime}(t)\right) \text { a.e. } \\
\text { on }\left\{t \in T: u_{m}(t)=0 \text { for some } m=1, \ldots, N\right\} \\
\left(f\left(t, u(t), u^{\prime}(t)\right)-\left(a\left(u^{\prime}(t)\right)\right)^{\prime}, u(t)\right)_{\mathbb{R}^{N}}=0 \text { for a.a. } t \in T \\
u=\left(u_{m}\right)_{m=1}^{N} \in C^{1}\left(T, \mathbb{R}^{N}\right), u(t) \geq 0 \text { for all } t \in T \\
u(0)=u(b)=0 .
\end{array}\right.
$$

\section{REFERENCES}

[1] S. Aizicovici, N. S. Papageorgiou, V. Staicu, Nonlinear nonconvex second order multivalued systems with maximal monotone terms, Pure Appl. Funct. Anal. to appear

[2] R. Bader, A topological fixed-point index theory for evolution inclusions, Z. Anal. Anwend. 20 (2001), 3-15. 
[3] H. Brézis, Opérateurs Maximaux Monotones et Semigroupes de Contractions dans les Espaces de Hilbert, North-Holland, Amsterdam, 1973.

[4] N. Dinculeanu, Vector Measures, Pergamon Press, Oxford, 1967.

[5] L. Erbe, W. Krawcewicz, Nonlinear boundary value problems for differential inclusions $y^{\prime \prime} \in f\left(t, y, y^{\prime}\right)$, Ann. Polon. Math. 54 (1991), 195-226.

[6] M. Frigon, Boundary and periodic value problems for systems of differential equations under Bernstein-Nagumo growth conditions, Differential Integral Equations, 8 (1995), 1789-1804.

[7] M. Frigon, E. Montoki, Systems of differential inclusions with maximal monotone terms, J. Math. Anal. Appl. 323 (2006), 1134-1151.

[8] L. Gasinski, N.S. Papageorgiou, Nonlinear Analysis, Chapman \&Hall/ CRC Press, Boca Raton, 2006.

[9] L. Gasinski, N.S. Papageorgiou, Exercices in Analysis. Part 2: Nonlinear Analysis, Springer, Heidelberg, 2016.

[10] N. Halidias, N.S. Papageorgiou, Existence and relaxation results for nonlinear second order multivalued boundary value problems in $\mathbb{R}^{N}$, J. Differential Equations, 147 (1998), 123-154.

[11] S. Hu, N.S. Papageorgiou, Handbook of Multivalued Analysis, Vol. I: Theory, Kluwer, Dordrecht, 1997.

[12] S. Kyritsi, N. Matzakos, N.S. Papageorgiou, Periodic solutions for strongly nonlinear second order differential inclusions, J. Differential Equations, 183 (2002), 279-302.

[13] R. Manasevich, J. Mawhin, Periodic solutions for nonlinear systems with $p$-Laplacian-like operators, J. Differential Equations, 145 (1998), 367-393.

[14] M. Marcus, V. Mizel, Absolute continuity on tracks and mappings of Sobolev spaces, Arch. Ration. Mech. Anal. 45 (1972), 294-320.

[15] D. Motreanu, V. Motreanu, N.S. Papageorgiou, Topological and Variational Methods with Applications to Nonlinear Boundary Value Problems, Springer, New York, 2014.

[16] T. Pruszko, Some applications of the topological degree theory to multivalued boundary value problems, Dissertationes Math. (Rozprawy Mat.) 229 (1984), 1-48.

[17] M. Zhang. Nonuniform nonresonance at the first eigenvalue of the $p$-Laplacian, Nonlinear Anal. 29 (1997), 41-51. 